\title{
ESTUDO EXPLORATÓRIO SOBRE A CONSULTA DE ENFERMAGEM
}

\author{
Ieda Barreira e Castro *
}

REFin/04

CASTRO, I. B. e - Estudo exploratório sobre a consulta de enfermagem, Rev. Bras. Enf., R.J., 28 : 76-94, 1975.

\section{I - INTRODUÇÃO}

A consulta de enfermagem, atividade institucionalizada no país recentemente, representa a culminação de um processo evolutivo do atendimento individual dos pacientes pela enfermeira de saúde pública.

$\mathrm{Na}$ verdade, ainda que esta atividade seja apropriada ao atendimento de qualquer paciente não hospitalizado, cabe à enfermeira de saúde pública o mérito de haver desenvolvido o procedimento a ponto de incorporá-lo definitivamente à prática da enfermagem. O fato decorre das características da implantação e da evolução da enfermagem no Brasil, que podemos classificar em quatro fases, como veremos no capítulo seguinte.

\section{II - FASES DA EVOLUÇAO DA ENFERMAGEM NO BRASIL}

A primelra fase vaí da criação da Escola Ana Neri, em 1923, sob os auspícios da saúde pública brasileira e com a ajuda norte-americana, à substituição, em 1929, das últimas visitadoras de higiene, do Departamento Nacional de Saúde Pública, por "enfermeiras diplomadas".

Logo vemos a importânâcia de dois grupos nos primórdios da profissão e que a influenciam até hoje: médicos brasileiros, que decidiram a implantação da enfermagem no País e enfermeiras americanas, que a implantaram, tanto na área de ensino como na de serviços.

A vinculação da primeira escola de enfermagem do país aos serviços de saúde pública, determinou o avanço espetacular da enfermagem de saúde pública que caracteriza esta fase inicial da profissão no Brasil. A enfermeira de saúde pública atuava diretamente junto ao público, nos centros de saúde e nos domicílios, sendo altamente valorizada sua função educativa junto à população.

A segunda fase estende-se até à segunda Grande Guerra e caracteriza-se como um período de transição e declínio. Os destinos da profissão passam às mãos das enfermeiras, é regulamentado o exercício da enfermagem no Bra-

- Auxillar de Enstono da Escola de Enfarmagem Ana Neri - URFJ. 
CASTRO, I. B. e - Estudo exploratório sobre a consulta de enfermagem, Rev. Bras. Enf.s R.J., 28 : 76-94, 1975.

sil* e criado o Ministério de Educação e Saúde. Sucessivas reformas administrativas terminam por prejudicar seriamente os resultados alcançados na primeira fase.

A organização de serviços de saúde pública nos Estados, pelas enfermeiras federais, determinada em 1938 e suspensa no ano seguinte, só pôde ser feita com o sacrifício do florescente serviço de enfermagem de saúde pública do Rio de Janeiro, então capital federal.

Esta tentativa provocou o reconhecimento da necessidade de adaptar as normas em uso na capital do país, à situação dos Estados, pois constatou-se logo a impossibilidade de se preencher os quadros de pessoal de enfermagem somente com enfermeiras e à conseqüerte deliberação de utilizar visitadoras sanitárias, em oposição à política adotada até então. Outra conseqüência foi o aumento do número de candidatas à Escola Ana Neri, pois o recrutamento passou a ser feito em todas as regiões do País.

A suspensão do trabalho dessas enfermeiras** que nos Estados treinavam as visitadoras, supervisionavam os serviços de enfermagem de saúde pública e assessoravam as autoridades, gerou uma crise nesses serviços e também nas escolas de enfermagem, que ficaram sem campos de prática. Pouco depois, uma outra reforma administrativa*** rebaixava 0 Serviço de Enfermeiras na estrutura administrativa da Prefeitura do Distrito Federal e lhe dava competência predominantemente normativa, o que acabou de tirar-lhe toda a autoridade e prestígio de que um dia gozara.

A terceira fase inicia-se com a criação do Serviço Especial de Saúde Pública (SESP), como expressão do esforço de guerra no Brasil. Também esse serviço de enfermagem foi organizado por enfermeiras americanas, estas, funcionárias do geverno de seu País, e que participavam ativamente da administração co serviço de enfermagem do SESP, nos primeiros anos.

Como este Serviço destinava-se exclusivamente ao interior do país e tinha objetivos a serem alcançados a curto prazo, teve que adaptar-se às possibilidades sócio-econômicas das nossas zonas rurais e com isto criou um novo modelo de saúde pública, mais compatível com a realidade brasileira. Sempre foi dado especial relevo, dentre as atribuições da enfermeira ao treinamento e à supervisão do pessoal auxiliar. Seus serviços surtiram um efeito de demonstração em todo o país; seus profissionais atuaram como agentes multiplicadores, ao colaborar com outras instituições, em nome do SESP ou levando consigo a experiência nele adquirida.

Nos anos após a guerra, a situação da enfermagem de saúde pública é precária, tanto nas escolas como nos serviços. Entrementes, os serviços de enfermagem dos hospitais lutavam por se firmar, principalmente nas entidades governamentais, uma vez que nos hospitais particulares, a presença da enfermeira era ainda mais discreta. A assistência de enfermagem a pacientes de ambulatórios, quer pertencentes a hospitais ou isolados, praticamente inexistia.

Este período foi de grande atividade, no que se refere às escolas de enfermagem, umas sendo criadas, outras equiparadas à "escola-padrão" ou incorporando-se a universidades.

A quarta fase iniciou-se em 1956, com a primeira pesquisa em enfermagem, $c$ Levantamento de Recursos e Necessidades de Enfermagem, de âmbito nacional, patrocinado pela Associação Brasileira

* Decreto n. 20.109/31.

** Decreto n. ${ }^{\circ}$ 1040/39.

*** Decreto n. $.^{\circ} 3171 / 41$. 
CASTRO, I. B. e - Estudo exploratório sobre a consuita de enîermagem, Rev. Bras. En£., R.J., 28 : 76-94, 1975.

de Enfermagem e financiado pela Fundação Rockfeller (que já havia colaborado para a criação da Escola Ana Neri). A atividade científica desenvolveu-se, notadamente, a partir de 1964, ano do XVI Congresso Brasileiro de Enfermagem, realizado em Salvador, Bahia, e que teve como tema oficial "Enfermagenı e Pesquisa". A partir de 1971, todos os Congressos têm apresentado o tema "Recentes Pesquisas em Enfermagem". Em 1959 foram abertos os primeiros cursos de saúde pública para enfermeiras, no Rio de Janeiro e São Paulo.

As reformas do ensino, de 1961 e 1966* apressaram a integração das escolas de enfermagem nas universidades brasileiras. O período caracteriza-se também pelo fortalecimento dos serviços de enfermagem nas estruturas de saúde do País e pela formação das primeiras equipes de planejamento de saúde, nas quais estava presente a enfermeira. Este último fato veio a contribuir decisivamente para o surgimento da consulta de enfermagem.

Já nos anos setenta surgiram os primeiros Cursos de Mestrado em Enfermagem ,no Rio de Janeiro e em São Paulc. Em 1973 a Escola Ana Neri comemorou o cinqüentenário de sua criação.

\section{III - ANTECEDENTES DA CONSULTA DE ENFERMAGEM NO BRASIL}

As raízes da atividade atualmente denominada consulta de enfermagem remontam ao início da profissão no Brasil. No manual preparado pelas enfermeiras americanas para as enfermeiras de saúde pública brasileiras, em 1925, na parte dedicada às doenças venéreas, constava como atribuição da enfermeira realizar entrevistas pós-clínicas com cada paciente nova, quando deveriam ser interpretados o diagnóstico e o trata-

* Leis n. ${ }^{\circ}$ S 4024/61 (Lei de Diretrizes e Bases) versitária). mento, a importância e os meios de prevenção da disseminação da doença.

Clementino Fraga (23), no Relatório Anual do Departamento Nacional de Saúde, dizia... "Durante o ano incrementou-se a ação e influência das enfermeiras de saúde pública nos consultórios de higiene infantil e a Inspetoria de Tuberculose adotou-as em dois dos seus dispensários, como educadoras dos casos novos matriculados, os quais, após a consulta, são dirigidos ao gabinete da enfermeira, para as recomendações e instruções indispensáveis, quanto à higiene individual e profilaxia.

LIMA (27), cita como atividade da enfermeira de saúde pública no programa de pré-natal, a entrevista com a gestante para a coleta de dados, preparo para a consulta médica, encaminhamento a serviços especializados, colheita de material para exames e aplicações de tratamentos prescritos. Defendia também que a enfermeira pudesse compreender os problemas gerais do todo familiar e que embora autoridades em serviço social considerassem essas atividades como privativas da assistente social, julgava que elas têm muito em comum com a enfermagem. Dizia ainda que com referência à parte educativa, a enfermeira é a pessoa mais indicada a executá-la.

Em 1958, a Comissão de Peritos em Enfermagem da OMS, reunida em Genebra, ao descrever as funções da enfermeira de saúde pública introduziu entre elas as funções médicas delegadas, cuja escolha deveria ficar a critério de cada país, de modo a atender às necessidades locais. A Fundação SESP iniciou experimentalmente $o$ atendimento pela enfermeira às gestantes sadias, ALVIM (2) estendido mais tarde a crianças sadias e, em 1966, incluía a atividade como de rotina em seu novo manual; ao mesmo

e Decretos Leis 53/66 e 252/67 (Reforma Uni- 
CASTRO. I. B. e - Erstudo exploratório sobre a consulta ảe enfermagem, Rev. Bras. Enf., R.J., 28 : 76-94, 1975.

tempo eram suspensas aquelas visitas de rotina às gestantes e crianças que tivessem finalidade de vigilância e estabelecido um sistema de prioridade.

Ainda no $2 .^{\circ}$ semestre de 1958 , realizaram-se no Recife, com um intervalo de menos de 2 meses, dois encontros que foram da maior importância para as enfermeiras dos dispensários de tuberculose. O primeiro foi o Seminário de Atualização em Saúde Pública, promovido pelo Serviço Especial de Saúde Pública (SESP), Departamento de Saúde Pública (DSP) do Estado, Departamento Nacional de Saúde (DNS), coordenado pela Chefe do Serviço de Enfermagem do SESP, Ermengarda de Faria Alvim. O segundo encontro foi a IV Reunião de Medicina Preventiva, promovida pela Sociedade de Higiene de Pernambuco, na cidade de Afogados de Ingazeiras. Os resuitados práticos imediatos desses encontros para os serviços de tuberculose, foram o irício da orientação do doente e de seus comunicantes, através de entrevistas pós-clínicas, o estabelecimento de um critério de prioridades para as visitas domiciliares e uma melhor articulação dos dispensários de tuberculose com os centros de saúde. Estas diretrizes foram adotadas anos mais tarde pelo então Setor de Enfermagem da Campanha Nacicnal Contra a Tuberculose e propostas às Secretarias de Saúde dos Estados da Federação, obtendo boa aceitação.

SANTOS (36) mostrava no XX Congresso Brasileiro de Enfermagem, realizado no Recife, em um painel denominado "A Realidade Sanitária do Nordeste", que segundo o tipo de assistência dada nas unidades sanitárias da FSESP, a gestante era atendida o mesmo número de vezes pelo médico e pela enfermeira e com igual previsão de tempo médio necessário à atividade.

Em 1967 BECKER (7), descrevendo a contribuição da Enfermagem na luta contra a tuberculose, alinhava, entre as ações relacionadas ao tratamento e controle do doente, entrevistas de cunho educativo. Nos anos seguintes o setor de Enfermagem da Campanha Nacional Contra a Tuberculose distribuiu para todos os Dispensários de Tuberculose que contavam com enfermeira, um Manual de Serviço, apresentando-o como a consolidação e ordenação das rotinas estabelecidas para os serviços de Enfermagem dos dispensários e já experimentadas na prática do trabalho diário, sendo pois o resultado da vivência do grupo de enfermeiras supervisoras do referido Setor.

Neste Manual (mimeografado), dezessete páginas são dedicadas a instruções para os diversos tipos de entrevista da enfermeira com o doente ou seus comunicantes. Estas entrevistas eram consideradas de suma importância para a continuidade do tratamento do doente, o que, por sua vez, era considerado a maior responsabilidade $d a$ enfermeira no serviço de tuberculose.

Após a entrevista realizada a cada comparecimento do doente, era movimentado um fichário de aprazamento, pelo qual controlava-se o comparecimento do paciente ao dispensário, colhiam-se os dados para o boletim estatístico do Serviço de Enfermagem, era feito o plano geral diário de visitas domiciliares, com prioridade para os faltosos e os novos, e também o entrosamento com o hospital de tuberculose. Quatorze páginas do Manual são dedicadas às instruções para a visitação domiciliar.

Em 1968, no segundo Curso de Planejamento de Saúde da Fundação Ensino Especializado de Saúde Pública (hoje Instituto Presidente Castello Branco), os profissionais participantes do mesmo concluíram ser o atendimento de enfermagem uma das atividades finais da unidade sanitária. Anteriormente esta atividade era considerada como um dos componentes da consulta médica. A par- 
CASTRO, I. B. e - Estudo exploratório sobre a consulta de enfermagem, Rev. Bras. Enf., R.J., $28: 76-94,1975$.

tir daí surgiu a denominação consulta de enfermagem, que vem difundindo-se desde então, particularmente no que se refere a gestantes ou crianças sadias.

\section{IV - DIFUSÃO DA CONSULTA DE ENFERMAGEM NO BRASIL}

Em fins de novembro de 1968, realizouse o Seminário Nacional sobre Currículo do Curso de Graduação em Enfermagem, organizado pela Escola de Enfermagem da Universidade de São Paulo (USP), que lhe serviu de sede, e patrocinado pela Organização Pan-americana de Saúde. Participaram do Seminário 126 enfermeiras, incluindo representantes de todas as Escolas de Enfermagem do País, outros especialistas em enfermagem e em educação e assessoras da OPAS/OMS.

O conteúdo do currículo foi estudado, considerando-se as funções atualmente desempenhadas pelas enfermeiras no Brasil. No campo da saúde da comunidade foi identificada a consulta de enfermagem como atividade final de maior importância e exclusiva da enfermeira, sem possibilidade de delegação.

Em 1969 a Seção Técnica de Enfermagem do Serviço Especial de Saúde de Araraquara já havia implantado a prática de consultas de enfermagem nas clínicas de saúde materna, saúde infantil e seguimento de algumas doenças crônicas de adultos.

SAYEG (37) ao descrever a Técnica de Programação Local - CENDES/OPAS, em trabalho do Departamento de Metodologia do Planejamento da FENSP, apresenta esta atividade como "final", mas ainda a denomina "atendimento de enfermagem". A atividade é conceituada como o atendimento "realizado, em geral para sadios ou para seguimento de tratamentos de alguns danos como, por exemplo, a tuberculose". Entretanto, ao explicar o preenchimento do modelo diz: "o volume das atividades é computado em número de consultas"...
CARVALHO (16), no mesmo ano, já cita entre as atribuições da enfermeira em um centro de saúde "dar consulta de enfermagem para gestantes e crianças".

COELHO, FARIAS \& MAGALHÃES (21), citam a experiência realizada pela Universidade Federal da Bahia, em colaboração com a Secretaria de Higiene e Saúde Pública do Estado em alguns campos de prática. Na distribuição das atividades dos membros da equipe de trabalho, cabe à enfermeira obstetra dar consultas à gestante, incluindo anamnese, exame obstétrico, elaboração do plano de assistência de enfermagem, requisições de exames de laboratório, controle da gestante patológica e seleção de casos para visitação domiciliar. A enfermeira pediatra compete dar consultas à criança sadia, incluindo anamnese, inspeção física, informação da mãe sobre o cuidado da criança, elaboração de um plano de assistência de enfermagem à criança, incluindo a observação do crescimento e desenvolvimento da criança, orientação dietética, imunizações e educação sanitária; encaminhamento da criança ao médico, quando necessário, controle da criança doente, através orientação após à consulta médica e seleção de casos para visitação domiciliar.

Em 1972 o Departamento de Recursos Humanos para a Saúde, do Instituto Presidente Castello Branco (ex-Escola Nacional de Saúde Pública), promoveu o encontro de enfermeiras do Ministério da Saúde, do Ministério da Educação e Cultura, do INPS, da Secretaria de Saúde do Estado da Guanabara e representantes da Associação Brasileira de Enfermagem, Seção Guanabara, que definiram os Padrões de Atribuições do Pessoal de Enfermagem. Como exemplos de tarefas típicas na prestação da assistência pela enfermeira, constam: "realizar entrevista para orientação, em serviços de proteção, de recuperação e de reabilitação da saúde e dar consulta de 
CASTRO, I. B. e - Estudo exploratório sobre a corsulta de enfermagem, Rev. Bras. Enf., R.J., $28: 76-94,1975$.

enfermagem para gestantes e crianças sadias, em programa materno-infantil".

Em 1972 o Setor de Enfermagem da Divisão Nacional de Organização Sanitária*, no documento elaborado como subsídio à Reunião de Ministros da Saúde realizada no Chile, incluiu a adoção da consulta de enfermagem como uma das três medidas gerais reconhecidas como importantes no tratamento de problemas de enfermagem. Esta adoção visaria a ampliação da assistência aos grupos materno-infantil e aos portadores de doença transmissível. Neste documento oficial a denominação consulta de enfermagem, refere-se indistintamente a.os dois grupos citados. Em 1971 a Fundação SESP, ao editar um manual para o Controle da Tuberculose, adotou as normas do então Serviço Nacional de Tuberculose e apresentou as instruções co Setor de Enfermagem da Campanha Nacional Contra a Tuberculose (CNCT), citadas no capítulo anterior, resumidas e adaptadas às características das unidades sanitárias da Fundação e manteve a denominação de entrevista, usada pelo Setor de Enfermagem da CNCT em época anterior à institucionalização da consulta de enfermagem. Do mesmo modo, no anteprojeto do seu novo Manual de Assistência MédicoSanitária Geral, elaborado em 1973, onde a Fundação SESP, pela primeira vez utiliza a denominação consulta de enfermagem, o faz somente em relação ao atendimento de gestantes e crianças sadias, diferenciando-a da orientação pós-clínica, que se segue à consulta médica, sendo as duas atividades computadas separadamente, para efeito de produção. O atendimento individual ao tuberculoso continua com a denominação de entrevista. Assim também, BORGES (8), ao preparar apostila para o Curso de Atualização em Tuberculose para
Enfermeiras, patrocinado pela Organização Pan-Americana de Saúde (OPAS) e realizada em Caracas, adaptou aquelas normas a situações mais gerais e apresentou-as como exemplo e como modelo das etapas da entrevista e da visita domiciliária ao tuberculoso, reforçando portanto, a mesma denominação. Para a FSESP, a consulta de enfermagem à gestante consiste na orientação sobre os recursos assistenciais da unidade, anamnese, verificação de sinais vitais, pedido de exames de laboratório. agendamento de consultas (médica ou de enfermagem), orientação quanto à sua saúde e à da criança, controle da vacinação antitetânica e de tratamento anti-sifilítico, encaminhamento para a visitação domiciliária, quando necessário, e orientação sobre o parto hospitalar, se for o caso. A consulta de enfermagem à criança consiste $\mathrm{n}_{\mathrm{a}}$ inspeção física para observação de anormalidades, verificação do peso e da temperatura, encaminhamento para imunizações, orientação da mãe quanto à prevenção de doenças, alimentação e cuidados higiênicos, encaminhamento à visitação domiciliária quando necessário. A FSESP estima que $40 \%$ do tempo da enfermeira da unidade deve ser gasto em consultas de enfermagem.

Em 1972 o Hospital de Clínicas de Porto Alegre (RS), iniciou um programa materno-infantil, no qual procurou dinamizar a assistência de enfermagem às crianças sadias, através de consultas de enfermagem que incluíam o exame clínico da criança, o controle de seu crescimento e desenvolvimento, o tratamento de problemas leves, a solicitação dos exames de laboratório de rotina, a orientação alimentar, as imunizações, a orientação quanto a hábitos e atitudes e suporte emocional. A avaliação dos oito primeiros meses do programa mos-

* Chefe do Setor de Enfermagem Judith Feitosa de Carvalho. 
CASTRO, I. B. e - Estudo exploratório sobre a consulta de enfermagem, Rev. Bras. Enf., R.J., 28 : 76-94, 1975.

trou que mais de $90 \%$ das mães compareceram às consultas subseqüentes aprazadas pela enfermeira e que menos de $10 \%$ das crianças foram encaminhadas à consulta médica.

Ao contrário das experiências anteriormente relatadas (de Araraquara, Salvador e Porto Alegre), todos ligados a Universidades, o Programa da Seção Materno-Infantil do Departamento Autônomo de Saúde Pública do Estado de Santa Catarina, para 1974, prevê a realização de consultas de enfermagem a gestantes e crianças, de menos de um ano, sadias, o que representa a aceitação da atividade por um órgão oficial de saúde pública.

Já em 1973 a Secretaria de Estado de Saúde do Estado do Ceará, oficializara a consulta de enfermagem em manual de normas e instruções.

\section{V - SituAÇÃo DA CONSUlTA DE ENFERMAGEM NA CIDADE DO RIO DE JANEIRO}

CASTRO (17) ao estudar a assistência de enfermagem a pacientes não hospitalizados, achou que 476 enfermeiras estão atualmente empenhadas diretamente nesta assistência, em serviços oficiais sediados na cidade, quer em ambulatórios de hospitais ou em ambulatórios isolados, quer em centros de saúde. Desses, $167(35 \%)$ atendem a pacientes em consultórios, quer sob a denominação de consulta ou de entrevista. Vemos que o número de enfermeiras dedicadas à atividade é marcadamente maior nos centros de saúde (onde $53,8 \%$ delas se dedica à atividade) do que nos ambulatórios oficiais $(9,8 \%)$ e nestes bem maior na área estadual $(18,9 \%)$ do que na federal $(5,4 \%)$. Mais da metade das enfermeiras de saúde pública dedica-se a esta atividade de assistência direta ao paciente, bem como mais de um terço do total de enfermeiras que assis- tem a pacientes não hospitalizados na cidade. (Tabela I)

A autora selecionou um grupo de enfermeiras, com vistas a uma sondagem de opinião, escolhendo-as devido aos altos cargos que ocupam (de chefia, de ensino ou de assessoria) e pelas qualidades de liderança que pressupóem as posições que galgaram. Assim as suas opiniões pessoais podem exercer grande influência tanto no âmbito interno das instituições onde trabalham, como junto às autoridades que deliberam sobre assuntos de saúde.

Das 16 enfermeiras entrevistadas, 10 representaram instituições assistenciais (sendo sete federais e três estaduais), cinco representam instituições educacionais (sendo três de cursos de graduação em enfermagem e duas de cursos de pósgraduação) e um representa a Associação Brasileira de Enfermagem na cidade. Entre os dez serviços representados, quatro estão ligados à assistência ambulatorial e seis à saúde pública.

Como resultado dessas entrevistas, desde logo ficou claro que a elaboração de normas escritas sobre como deve a enfermeira assistir ao paciente não hospitalizado não é uma necessidade reconhecida por todas as instituições que, de algum modo, têm o poder de decisão sobre o assunto e nem mesmo por todas as que prestam essa assistência à população, pois apenas a metade as possui.

$\mathrm{O}$ atendimento individual dos pacientes, o seu ensino sobre assuntos relacionados à saúde e à prestação de cuidados de enfermagem a certos pacientes, são atividades reconhecidas pelas instituições como própria da enfermeira que assiste a pacientes não hospitalizados. A visita domiciliária só foi reconhecida por cerca da metade delas.

Na parte referente à opinião das entrevistadas sobre quais deveriam ser as atribuições das enfermeiras junto aos pacientes não hospitalizados, vemos que 
CASTRO, I. B. e - Estudo exploratório sobre, a consulta de enfermagem, Rev. Bras. Enf., R.J., 28 : 76-94, 1975.

nenhuma atividade foi apontada unanimemente. A mais citada foi o desenvolvimento de atividades educativas com a clientela. A consulta e/ou entrevista vem a seguir (apenas 2 representantes de instituições educacionais e/ou culturais $(1 / 3)$ citaram a consulta e/ou entrevista de enfermagem como atividade própria da enfermeira). A visitação domiciliária e os cuidados de enfermagem a casos agudos obtiveram igual citação ficando em terceiro lugar. $O$ atendimento individual (estrevista e/ou consulta) foi mais valorizado pelos serviços do que por suas representantes (Tabela II) .

A estrutura da consulta de enfermagem foi esclarecida pelo reconhecimento ou não, por 10 das enfermeiras entrevistadas, das diversas fases da metodologia do processo de enfermagem, como componentes da consulta de enfermagem, como julgam que é executada nos serviços da instituição a que pertencem. Vemos que todas as entrevistadas foram unânimes em reconhecer que a atividade, na prática, consiste na coleta de dados (feita no prontuário e por informação, observação ou exame do paciente), seguida imediatamente da assistência de enfermagem. As fases de diagnóstico de enfermagem, plano assistencial de enfermagem e prognóstico não foram reconhecidas. A atividade consuita de enfermagem, como é executada, não implica na utilização de uma metodologia e portanto não difere de uma entrevista de enfermagem. Em relação à assistência de enfermagem todos reconheceram como componentes da atividade: a seleção das necessidades prioritárias da situação apresentada, a orientação e 0 aconselhamento em relação à saúde, diagnóstico e tratamento médicos, regime de vida, assistência oferecida pela instituição e papel da equipe e finalmente o aprazamento dos próximos comparecimentos ou visitas (Tabela III).

Das 16 entrevistadas, 14 se pronuncia- ram favoravelmente em relação à consulta de enfermagem e 2 desfavoravelmente ou com restrições. Entre as razões afirmativas, a mais citada foi a de que "é um procedimento adequado às atuais funções da enfermeira na assistência ao paciente não hospitalizado", seguida por "permite a melhor utilização do médico e/ou do hospital" e por "favorece a melhoria qualitativa e/ou quantitativa da assistência de enfermagem".

As razões negativas ou restritivas, foram: "não é atividade prioritária, só deve ser feita havendo disponibilidade de horas-enfermeiras"; "apresenta menor rendimento do que as atividades em grupo" e "não há necessidade de delegação de funções médicas, em virtude de proporção numérica inversa médicosenfermeiras". De modo geral as enfermeiras mostram-se favoráveis à consulta como atividade específica da enfermeira que atende a pacientes não hospitalizados. Os argumentos contra a consulta parecem demonstrar a identificação da consulta como atividade meramente educativa e como função médica delegada (Tabsla IV).

A adaptação da enfermeira à função e à preparação da equipe de saúde e da comunidade foram consideradas necessárias à introdução da consulta além do treinamento das enfermeiras para a atividade (que ficou em primeiro lugar) e sua melhor seleção. O exposto parece indicar que as enfermeiras entrevistadas julgam que as enfermeiras encarregadas da assistência aos pacientes não hospitalizados não estão aptas a dar consultas e desconfiam da reação que a equipe e a própria clientela teriam à introdução da atividade (Tabela V).

A consulta de enfermagem foi caracterizada como atividade de deliberação racional, baseada em metodologia própria. Inclui o exame físico do paciente, estabelece relações de confiança, tem objetivos complexos, imediatos e mediatos, com ênfase na prevenção. Consti- 
CASTRO, I. B. e - Ezstudo exploratório sobre a consulta de enfermagem, Rev. Bras. Enf., R.J., 28 : 76-94, 1975.

tui-se em uma atividade final, isto é, dispensada diretamente ao paciente. A entrevista, por sua vez, é intuitiva ou, ao contrário, baseada em roteiros préestabelecidos, tem objetivos mais simples e imediatos, desenvolve-se principalmente por meio do interrogatório do paciente e é complementar à consulta méciica (Tabela VI).

A maioria das enfermeiras entrevistadas julga que a consulta de enfermagem deve ser prerrogativa da enfermeira, enquanto um quarto julga que a atividade pode ser delegada a outras categorias da equipe de enfermagem (Tabela VII).

A atividade consulta de enfermagem é aceita a nível institucional e formalmente valorizada pelas enfermeiras representantes, que entretanto, não a lembraram espontaneamente com a mesma freqüência, o que parece demonstrar que a importância da consulta de enfermagem foi por elas compreendida, mas não assimilada como valor. Talvez por isto a atividade só seja executada nos serviços de um terço das instituições representadas (Tabela VIII).

De modo geral os tuberculosos, as gestantes e as crianças são atendidos individualmente pelas enfermeiras, tanto em ambulatórios como em centros de saúde. O atendimento dos pacientes tuberculosos é denominado entrevista e o das gestantes e crianças é chamado consulta de enfermagem, havendo uniformidade nessas denominações entre as diversas entidades representadas (Tabela IX).

E interessante notar que as clientelas aterdidas em consuitórios de enfermagem, quer sob a denominação de "entrevistas de enfermagem", como no caso dos tuberculosos, quer sob a denominação de "consulta de enfermagem", como no caso de gestantes e crianças (e que na realidade da prática atual não diferem muito), já o eram no início da enfermagem de saúde pública do Rio de J́aneiro.

\section{VI - DISCUSSÃO}

A visitação domiciliária, que se pretendeu durante algum tempo considerar como função característica da enfermeira de saúde pública, no Brasil mostrouse inviável. O número insuficiente de enfermeiras de saúde pública, o alto custo da atividade e o seu valor relativo ccmo atividade isolada, fizeram com que esta atividade passasse a ser quase completamente delegada a pessoal auxiliar e posteriormente reservada a casos selecionados, segundo um critério de prioridades, e como complemento a outras atividades desenvolvidas pela enfermagem.

A consulta de enfermagem surgiu em nosso País em 1958, em decorrência de um processo que tem suas raízes nos primórdios da profissão no Brasil.

A entrevista pós-clínica foi precursora da consulta, aquela a nível de prevencão terciária* e esta como "função médica delegada", a nivel de prevenção primária". Esta delegação foi motivada pela necessidade de uma melhor divisão do trabalho entre o médico e a enfermeira, a fim de liberar o médico para os cascs que demandam exames médicos $n$ fazendo do atendimento pela enfermagem um substituto para a visita domiciliária, que deixara de ser a atividale básica da enfermagem de saúde pública. Esta "delegação de funções" cvoiuiu progressivamente até caracterizar-se como uma "transferência de funcões" do médico para a enfermeira. O faio deveu-se à evolução das duas profissõ's e constitui uma expansão do papel da enfermeira. Entretanto, a consulta de enfermagem, antes de ser uma função inteiramente nova, na realidade

* Conforme classificação de CLARK \& LEAVELI (1958). 
CASTRo, I. B. e - Estudo exploratório sobre a consulta de enfermagem, Rev. Bras. Enf.y R.J., 23 : $76-94,1975$.

consiste na reunião de várias tarefas, em uma só atividade. Como diz ROGER' (35) as assim chamadas "novas funções" da enfermeira, na verdade integram a prática de enfermagem há décadas $\mathrm{e}$ realmente "nenhuma profissão delega nada a outra". Os aspectos novos a serem considerados na atividade são a sua estrutura orgânica e o reconhecimento da responsabilidade direta da enfermeira em relação aos seus assistidos. O primeiro suscita um novo problema, qual seja o da metodologia, implicando a consulta no desenvolvimento do processo de enfermagem e não sendo neste caso passível de delegação.

\section{VII - CONCLUSÓES}

1. A consulta de enfermagem é atividade institucionalizada na área de ensi- no como na de serviços e nesta tanto na esfera federal como na estadual.

2. A consulta de enfermagem configura-se atualmente como a atividade característica da enfermeira que assiste os pacientes nas unidades sanitárias e esta tendência vem estendendo-se aos ambulatórios de hospitais ou isolados.

3. A "entrevista de enfermagem" não difere na prática da consulta de enfermagem, devendo a primeira ser englobada na segunda denominação.

4. É imperativo o desenvolvimento de uma metodologia da consulta de enfermagem, que a caracterize como prerrogativa da enfermeira e a coloque em posição apropriada para o desempenho de seu papel na equipe de saúde junto ao paciente não hospitalizado. 


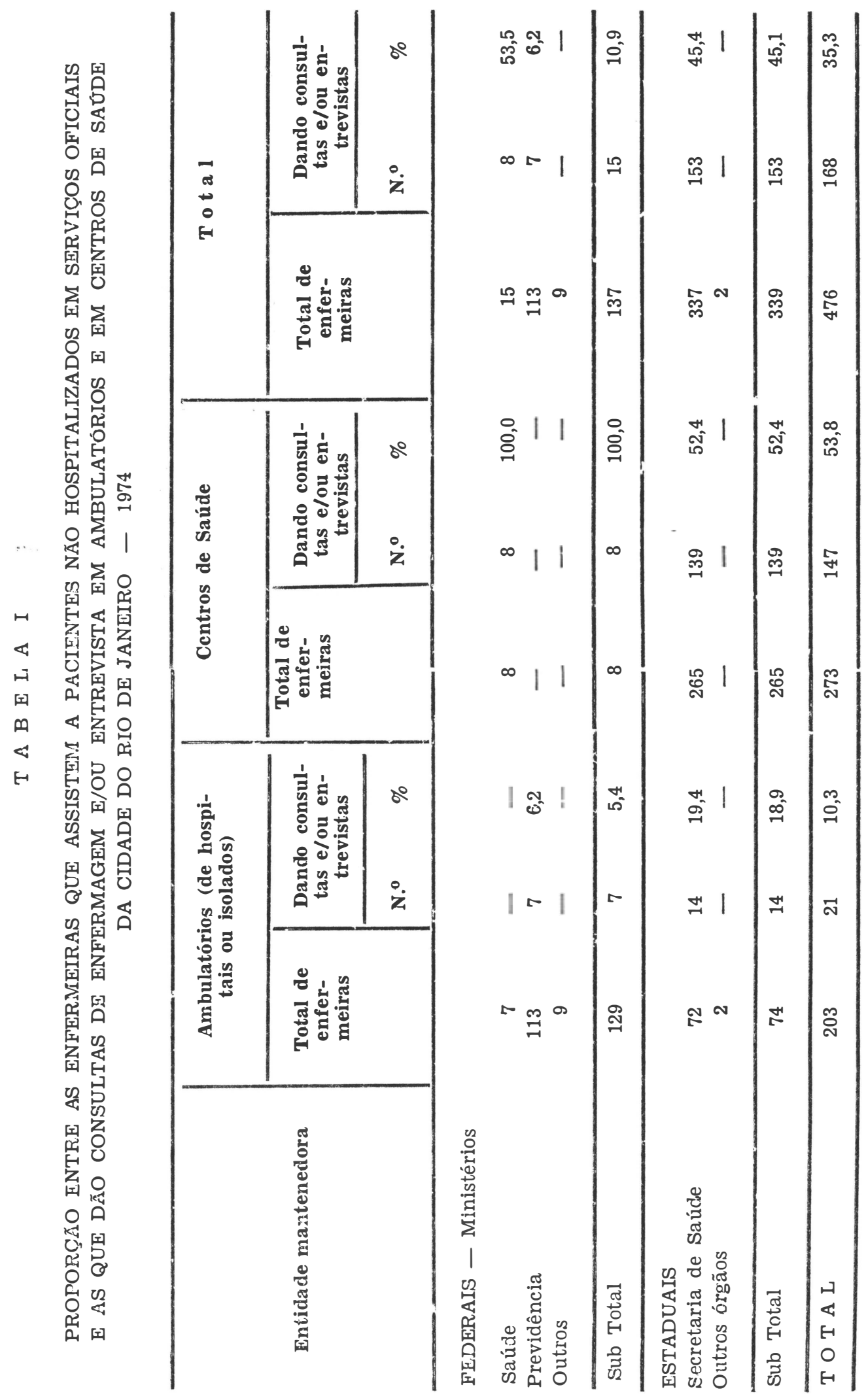


INFORMAÇÓES DAS ENTREVISTADAS DE 12 INSTITUIÇÕES* QUE TÊM NORMAS (ESCRITAS OU NAO) SOBRE AS ATRIBUIÇÓES DAS ENFERMEIRAS JUN-. TO AOS PACIENTES NAO HOSPITALIZADOS E OPINIAO DE 16 ENTREVISTADOS SOBRE QUAIS DEVERIAM SER ESSAS ATRIBUICOOES

\begin{tabular}{|c|c|c|c|c|}
\hline \multirow{2}{*}{ Atribuições } & \multicolumn{2}{|c|}{$\begin{array}{l}\text { Norma das } \\
\text { instituições }\end{array}$} & \multicolumn{2}{|c|}{$\begin{array}{l}\text { Opinião dos } \\
\text { entrevistados }\end{array}$} \\
\hline & N.o & $\mathbf{P}$ & N.o & $\mathbf{P}$ \\
\hline 1. Consulta e/ou entrevistas ........... & 12 & 10,0 & 9 & 5,6 \\
\hline 2. Atividades educativas com a clientela & 10 & 8,4 & 13 & 8,1 \\
\hline 3. Visitação domiciliar ............... & 7 & 5,8 & 6 & 3,8 \\
\hline $\begin{array}{l}\text { 4. Cuidados de enfermagem a casos } \\
\text { agudos } \ldots \ldots \ldots \ldots \ldots \ldots \ldots \ldots \ldots \ldots \ldots\end{array}$ & 8 & 6,7 & 6 & 3,8 \\
\hline 5. Outros atendimentos à clientela .... & 2 & 1,6 & - & - \\
\hline 6. Controle de comunicantes .......... & 1 & 0,8 & 2 & 1,2 \\
\hline
\end{tabular}

$$
\mathrm{P}=\text { probabilicade }-\frac{\mathrm{N}^{\mathrm{O}} \text { de casos favoráveis }}{\mathrm{N}^{\circ}{ }^{\circ} \text { de casos possíveis }} \times 10
$$

* 10 assistenciais, 1 de pós-graduação e 1 cultural 


\section{R a z õ es a pont a d a s}

N.o

$\mathbf{P}$

1. Afirmativas

1.1 - a enfermeira está capacitada para a promoção e a proteção da saúde...$\ldots \ldots \ldots \ldots \ldots \ldots$.

1.2 - é um procedimento adequado às atuais funções da enfermeira na assistência ao paciente não hospitalizado $\ldots \ldots \ldots \ldots \ldots \ldots \ldots \ldots \ldots \ldots$

7

1.3 - favorece a melhoria dal assistência de enfer-

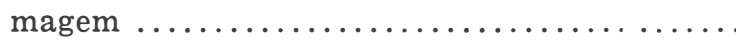

1.4 - permite a melhor utilização do médico e/cu do

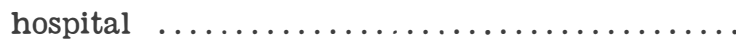

1.5 - desenvolve habilidades específicas de enfermagem

1.6 - eleva c "status" profissional da enfermeira ...

2. Negativas ou restritivas

2.1 - não é atividade prioritária, só deve ser feita havendo disponibilidace de horas-enfermeiras .

2

2.2 - apresenta menor rendimento que as atividades

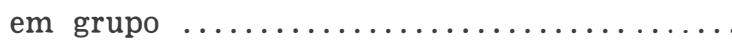

1

2.3 - não há necessidade de delegação de funções médicas,, em virtude da proporção inversa médicos-enfermeiras $\ldots \ldots \ldots \ldots \ldots \ldots \ldots \ldots \ldots$

1

0,6 
CONDIÇÕES CONSIDERADAS NECESSÁRIAS PELAS 16 ENFERMEIRAS ENTREVISTADAS PARA A INTRODUÇÃO DA ATIVIDADE CONSULTA DE ENFERMAGEM NESTA ASSISTÊNCIA

\section{Co n d i ço es ne cessárias}

N.o

10

6,2

1.1 - Treinamento

1.2 - Melhor seleção das das enfermeiras .......

4

2,5

1.3 Melhor utilização das horas-enfermeiras ......

2

1,2

$1.4-$ Mais pessoal de enfermagem $\ldots \ldots \ldots \ldots \ldots$

2

1,2

1.5 - Mais recursos materiais

1

0,6

2. Aspectos institucionais

2.1 - Filosofia e/ou política da instituição

2

1,2

2.2 - Instrumentos legais para a reformulação dos serviços $\ldots \ldots \ldots \ldots \ldots \ldots \ldots \ldots \ldots \ldots \ldots \ldots$

3

1,8

2.3 - Melhor sistema de ccordenação e comunicação

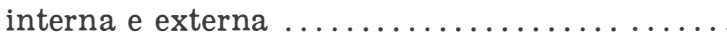

2.4 - Definição de papéis, funções e atribuições ....

2

1,2

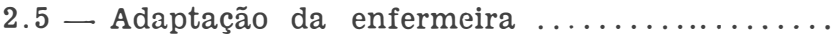

4

2,5

3

1,8

2.6 - Preparação da equipe e da comunidade

5

3,1

\footnotetext{
$P=$ Probabilidade $=\frac{\text { N. }^{\text {de casos favoráveis }}}{\text { N. }^{\circ} \text { de casos possíveis }} \times 10$
} 


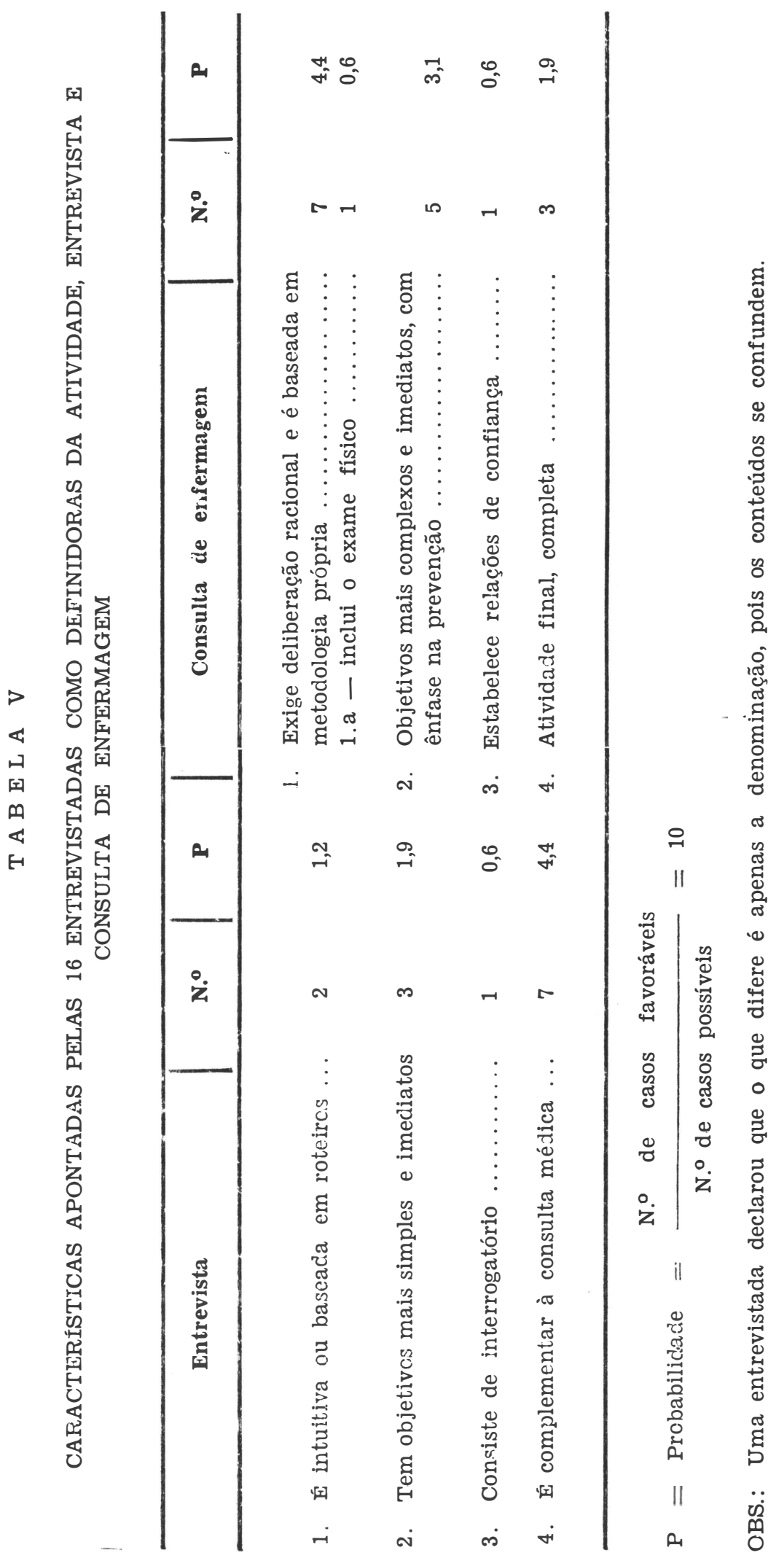


T A B E L A VI

ELEMENTOS DA EQUIPE DE ENFERMAGEM HABILITADOS A DAR CONSULTAS

DE ENFERMAGEM, CONFORME A OPINIÃO DAS ENFERMEIRAS ENTREVISTADAS, SEGUNDO SUA ÁREA DE TRABALHO

\begin{tabular}{|c|c|c|}
\hline Categorias & $\mathbf{N} \cdot \mathbf{0}$ & $\mathbf{P}$ \\
\hline 1. Só a enfermeira & 12 & 7,5 \\
\hline 2. Enfermeira, técnica de Enfermagem .... & 1 & 0,7 \\
\hline $\begin{array}{l}\text { 3. Enfermeira, Auxiliar de Enfermagem e.'ou Visitadora } \\
\text { Sanitária } \ldots \ldots \ldots \ldots \ldots \ldots \ldots \ldots \ldots \ldots \ldots \ldots \ldots \ldots\end{array}$ & 3 & 1,8 \\
\hline
\end{tabular}

$P=$ Probabilidade $=\frac{\text { N. }^{\circ} \text { de casos favoráveis }}{\text { N.० de casos possíveis }} \times 10$

T A B E L A VII

COMPARAÇÃO ENTRE A NORMA DA INSTITUIÇÃO, A OPINIÃO ESPONTÅNEA DA ENFERMEIRA, SUA OPINIÃO QUANDO INTERROGADA F A EXECUÇÃO DA ATIVIDADE CONSULTA DE ENFERMAGEM E/OU ENTREVISTA NA ROTINA DE TRABALHO DOS SERVIÇOS

\section{A $t$ ividade prese n t e}

N.o

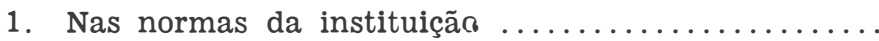
12

7,5

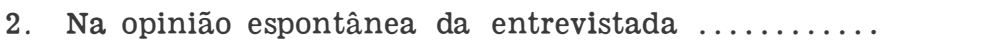

3. Na opinião da entrevistada quando interrogada .... 14

4. Na rotina de trabalho dos serviços de enfermagem

6

5,6

48,7

3,8

$\mathrm{P}=$ Probabilidade $=\frac{\mathrm{N}{ }^{\circ} \text { de casos favoráveis }}{\mathrm{N} \cdot{ }^{\circ} \text { cel casos possíveis }} \times 10$


CLIENTEIA ATENDIDA PELA ENFERMEIRA, EM CONSULTAS E/OU ENTREVISTAS, NOS SERVIÇOS DE ADMINISTRAÇÃO DIRETA DAS 7 ENTIDADES REPRESENTADAS NO INQUÉRITO QUE OS POSSUEM E DENOMINACQÃO DADA À ATIVIDADE:

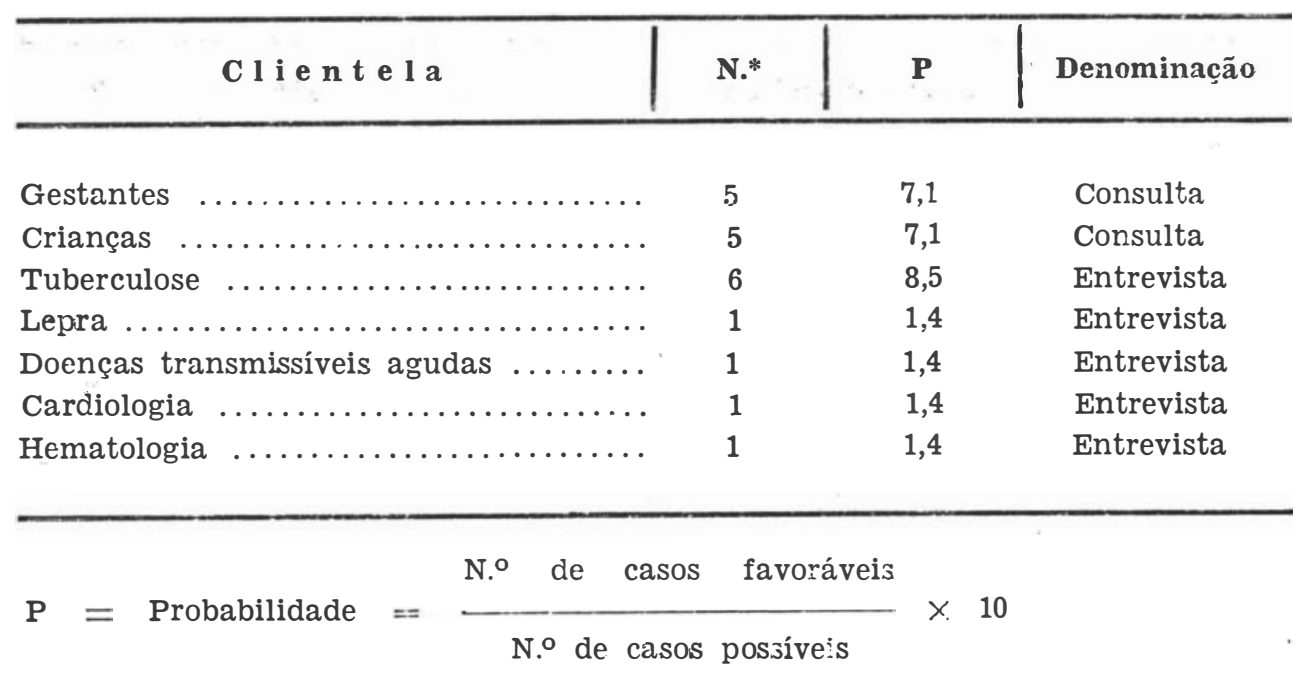

BIBLIOGRAFIA

1. ALCÂNTARA, Glete de - Novas tendências da educação da enfermagem. A educação de enfermagem entre 1923 e 1961. Rev. Bras. Fnf, Rio de Janeiro, 17 (5) : 335-45, out, 1964.

2. ALVIM, Ermengarda de Faria - Funç̃es delegadas ao Serviço de Fnfermagem. Seminário (S.N.T.) (mimeografado).

3. ALVIM,Ermengarda de Faria - Quinze anos de enfermagem no SESP Rev. Bras. Enf., Rjo de Janeiro, 12 (2) junho, 1959.

4. ASSOCIAC̣ÃO BRASILEIRA DE ENFERMAGEM - Levantamento de recursos e necessidades de enfermagem. Rio de Janeiro, 1955 (mimeografado).

5. BARBOSA, Laís Maria Horta \& DANTAS, Maria do Carmo - Histórico da Escola de Enfermagem "Ana Neri". Universidade Federal do Rio de Janeiro (antiga Universiclade do Brasil). Rev. Bras. Enf, Rio de Janeiro, 19 (2 e 3): 161-65, abr.! jun., 1966.
6. BASTOS, M.C. de Erito - Treinamento de pessoal de Saúde Pública 23 anos de experiência da Fundação SESP 1942-1965. Rio de Janeiro, F. SESP, 1966.

7. BECKER, Raimunda - Contribuição da enfermagem na luta contra a Tuberculose. Rev. Bras. Enf., Rio de Janeirc, 20 (4) : 348-363, ago. 1937.

8. EORGES, Maria Valderez - Enfer'meria en el tratamiento ambulatório del paciente tubercu!oso. Notas para el curso de atualización en tuberculosis para enfermeras. Caracas, 1973.

9. BRASIL. CAMPANHA NACIONAL CONTRA A TUBERCULOSE - Manual do Serviço de Enfermagem no Dispensário. Rio de Janeiro, Serviço Nacional de Tuberculose, 127 p. (mimeografado) 1969.

10. BRASIL. DEPARTAMENTO NACIONAL DE SAÚDE PÚBLICA - Manual preparado para as enfermeiras de saúde pública do Serviço de Enfermeiras pela Missão Téc- 
CASTRO, I. B. e - Eistudo exploratório sobre a consulta de enfermagem, Rev. Bras. Enf., R.J., 28 : 76-94, 1975.

nica de Enfermeiras Americanas e aprovado por Dr. Raul Leitão da Cunha, Diretor Geral em exercício do DNSP. Rio de Janeiro, 1925.

11. RRASIL. DIVISÃO NACIONAL DE ORGANIZAÇÃO SANITARIA. SETOR DE ENFERMAGEM - DOcumento elaborado para a Reunião. de Ministrcs no Chile, 1972. (Mimeografado).

12. BRASIL. FUNDAÇ.̃̃O SERVIÇOS DE SAƯDE PÍ́BLICA - Controle da Tuberculose - Normas e instruções, Rio de Janeiro p: 23-132, 1971.

13. BRASIL. FUNDACÃO SERVICOS DE SAÚDF PÚBLICA - Assistência médico-sanitária geral - No"mas e instrucões (antiprojeto) Fio de Janeiro, 1973.

14. BRASIL. FUNDAÇÃO SERV'ICCOS DE SAÚDE PÍBLICA - Enfermagem, Jegislnção e assuntos correlatos, $3 .^{a}$ ed., Rio de Janeiro, 1974.

15. BRASIL. INSTITUTO PRESIIUENTE CASTILO BRANCO. DEPARTAMENTO DE RECURS(JS HUMANOS PARA SAŨRE - Padróes de atribuicõos do pessoal de enfermagem, 1972 (mimeografado).

16. CARVALHO, Judith Feitosa - Organização de serviços de ənfermagem em Saúde Pública. Rev. Bras. Enf. Rio de Jantiro, $22(1,2$ e 3$)$ : 19-26, jan/jun, 1669.

17. CASTRO, Teda Rarreira \& BEZERRA, Stella do Valle - Fnfermagem nos dispensários de tuberculose do Recife. Rev. Bras. Enf., Rio de Janeiro, 15 (6) : 569-74, dez., 1961.

18. CAstro, Ieda Barreira e - Aspecto; crítices do desempenho de funções próprias da enfermeiril na assistência ao paciente não hospitalizado. Dissertação de Mestrado apresentada à Escola de Enfermagem Ana Neri Universidade Federal do Rio de Janeiro, :975.

19. CEARÁ. Secretaria de Saúde. Manual de Fnfermagem -- Normas e Ins truçōes - Fortaleza, 84 p., 1973.
20. CLAFK, E. G. \&: LEAVELL, H. R. I.evels of application of preventive medicine. In - Preventive medicine for the dactor in his community 2 ed. N. York, Mcciraw - Hill Book Co., 1958.

21. COELHO, Célia Galvão Dias; e tal - O Papel da Enfermeira na assistência materno-infantil. Anais do Seminário sobre: $O$ Ensino da proteção à saúde materno infantil, em função das necessidades da comunidade. 23 a 26 de novembro. Salvador, Associação Brasileira de Escolas Médicas, 1970.

22. DOURADO, Haydée Guarnais - Resenha histórica da enfermagem no Brasil. Rio de Janeiro, Divisão de Organização Sanitária, 2950.

23. FRAGA, Clementino - Introdução ao Relatório dos Serviços do Departamento Nacional de Saúde Pública. Arquivos de Higiene. DNSP, Rio de Janeiro, 2 (1) : 214-215, maio, 1928.

24. JORG' Mirabel S. Ferreira - Problemas de enfermagem de saúde pública no Distrito Federal. Anais de Enfermagem, São Paulo, 16 (22) : 6163, jan. 1947.

25. I_ENINGTOM, Beatrice \& TEIXEIRA, Gcorgette - O papel do Serviço de Enfermagem na organização hospitalar Rev. Bras. Enf, Rio de Janeiro 8 (3) : 189-199, set, 1955.

26. LIMA, Izaura Parbosa - Aspecto da situação da Enfermagem no Brasil. Rio de Janeiro. Divisão de Organização Sanitária, 1950.

27. LIMA, Izaura Barbosa - Resenha hisEquipe de Saúde Pública. Rio de Janeiro, Divisão de Organizaçāo Sanitária, 1956.

28. IIMA. Izaura Barbosa. Resenha histórica da enfermagem de saúde pública do Ministério da Saúde (19211972) Rio de Janeiro 1973 - (Datilografado).

29. LOPEZ, Juana L. de la Peña - A enfermeira de saúle pública. Contribuicão ao estudo de suas funcões S. Paulo, 1971 (tese). 
CASTRO, I. B. e - Estudo exploratório sobre a consulta de enfermagem, Rev. Bras. Enf., R.J., 28 : 76-94, 1975.

30. MATOS, Adalgisa Vieira - Organização do Serviço de Enfermagem da Fundação Serviço Especial de Saúde Pública. Rev. Bras. Enf. Rio de Janeiro, 24 (1 e 2) : 40 janeiro/ mar., 1971.

31. MEDEIROS, Maria Marlene B. \& FLSEM, Ingrid - Organização do Serviço de Enfermagem de Saúde Fública do Departamento Autônomo de Saúde Pública da Secretaria de Estado de Saúde de Santa Catarina, Rev. Bras. Fnf., Rio de Janeiro, 2y (4) : 480-498, 1 y74.

32. MUXFELD, Lea; e tal - O papel na assistência à criança sadia. Programa Materno-Infantil do Hospital das Clínicas de Porto Alegre, 1972.

33. ORGANIZAÇÃO MUNDIAL DA SAÚDE. COMITÉ DE EXPERTOS DE A OMS EN ENFERMERIA. Enfermeria de Salud Publica Ginebra, 1975 (Série de informes técnicos, 167. - 4..$^{\circ}$ informe.)
34. PINHEIRO, Maria Rosa de Souza - A enfermagem no Brasil e em $\mathbf{S}$. Paulo. Rev. Bras. Enf., Rio de Janeiro, 15 (5) out. 1962.

35. ROGERS, M. - Nursing: to be or not to be ? Nursing Out look 20 (1) 42-46 jan, 1972.

36. SANTOS, Isabel - Participação da enfermagem na satisîação da demanda nos serviço de saúde. Rev. Bras: Enf. 21 (5): out 1968.

37. SAYEG, Maric - Modelos, normas e procedimentos operacionais. Técnica de Programação local CENDES/OPAS. Rio de Janeiro, Fundação de Recursos Humanos para a Saúde, 1969 . (mimeografad o).

38. SEMINARIO NACIONAL SOBRE CUR. RÍCULO DO CURSO DE GRADUAÇÃO EM ENFERMAGEM, Sãc Paulo, março, 1969. São Paulo. Universidade /OPAS, 1969. 